\title{
Musculoskeletal imaging: current and future trends
}

\author{
Hassan Douis • Steven L. J. James • A. Mark Davies
}

Received: 20 September 2010 / Accepted: 14 October 2010 /Published online: 22 December 2010

(C) European Society of Radiology 2010

\begin{abstract}
Advances in imaging technology and the increasing role of interventional procedures in musculoskeletal imaging have continued to stimulate research over recent years. This review summarises some recent articles on musculoskeletal radiology topics and looks forward to potential future developments in this exciting sub-speciality.
\end{abstract}

Keywords Musculoskeletal imaging · Trends · MRI .

Vertebroplasty $\cdot$ Cost-effectiveness

\section{Introduction}

Musculoskeletal imaging has benefitted tremendously from an increasing body of work investigating new imaging techniques and the application of established techniques in novel fields. In addition, interventional musculoskeletal radiology has become an enthusiastically researched topic. Between January 2008 and July 2010, 111 papers have been published in European Radiology on musculoskeletal imaging. These include: 91 original articles, 11 review articles and 9 case reports (Table 1). Given the exciting developments in this field, we wish to highlight the key advances in musculoskeletal imaging over this period that have been published in this journal and speculate on possible future trends. We include some spinal imaging and intervention in this review though acknowledge some overlap with the sub-speciality of neuro-radiology exists.

\footnotetext{
H. Douis $\cdot$ S. L. J. James $\cdot$ A. M. Davies $(\bowtie)$

Department of Radiology,

The Royal Orthopaedic Hospital NHS Foundation Trust,

Bristol Road South, Northfield,

Birmingham, UK B31 2AP

e-mail: Wendy.Turner@roh.nhs.uk
}

\section{Imaging of tumours and tumour-like lesions}

The crucial role of radiologists in the diagnosis, staging and increasingly in the treatment of selected bone tumours has been reflected by the number of articles published in this journal about this topic in recent years. Conventionally the diagnosis and staging of musculoskeletal tumours involves radiographs, MRI, CT and skeletal scintigraphy. The emergence of PET/CT has revolutionized the staging of a wide range of solid tumours. However, little is known about the role of PET/CT in the staging of musculoskeletal malignancies. Lakkaraju et al., reviewed the literature for the evidence of PET and PET/CT in the evaluation and staging of primary musculoskeletal tumours [1] (Fig. 1). Their review reveals that the role of PET/CT in primary musculoskeletal tumours remains poorly understood and future work in this area is needed to help better define its use.

In the article by Alyas and Saifuddin [2], the authors have focused on a well recognised MRI appearance (fluidfluid levels) and used this sign in an attempt to further characterise whether a benign or malignant process is being encountered. They retrospectively evaluated 214 patients with fluid-fluid levels and assessed the diagnostic relevance of relative signal intensity of the superior and inferior layer of fluid-fluid levels. They found that low/high signal on T1WI was significantly associated with benign disease whilst, high/low signal intensity on T1WI was more commonly associated with malignant lesions. In contrast, the high/low signal intensity pattern on T2WI was identified as a non-specific pattern. These findings may therefore help to differentiate benign versus malignant bone tumours.

Singh and co-workers [3] performed a multicentre retrospective analysis of patellar lesions in four European 
Table 1 Table illustrating the most commonly encountered musculoskeletal topics published in European Radiology from January 2008 to July 2010

\begin{tabular}{lc}
\hline Topics published in European Radiology & $\begin{array}{l}\text { Number of articles } \\
\text { published (\%) }\end{array}$ \\
\hline Tumours and tumour-like lesions & $23(20.7 \%)$ \\
Musculoskeletal ultrasound & $16(14.4 \%)$ \\
Cartilage imaging and imaging of the knee & $16(14.4 \%)$ \\
Myeloma, bone marrow and skeletal & $11(10 \%)$ \\
$\quad$ metastases & $10(9 \%)$ \\
Vertebroplasty and other spinal & \\
interventional procedures & $6(5.4 \%)$ \\
Imaging of the shoulder & $6(5.4 \%)$ \\
Other topics & $23(20.7 \%)$ \\
Total & 111 \\
\hline
\end{tabular}

bone tumour registries producing the largest published series of primary patellar lesions. The authors identified 59 lesions in the patella of which: $46 \%$ were non-neoplastic, $39 \%$ were benign and $15 \%$ were malignant. The most common benign lesions were giant cell tumours. Lesions in patients younger than 40 years of age included giant cell tumour, chondroblastoma, aneurysmal bone cyst, osteomyelitis, osteoid osteoma and solitary bone cyst. In patients older than 40 years, intra-osseous gout, metastasis and intra-osseous ganglion were common lesions. This study has therefore enhanced our understanding of the nature of patellar lesions.

Radiologically-guided interventional treatment for certain bone tumours has gained in popularity. Percutaneous interventional therapy for instance has established itself as the treatment of choice for osteoid-osteoma. However, radiologically-guided treatment of osteoid-osteoma of the hands and feet has been thought difficult due to the close proximity of neurovascular bundles and skin, and the small size of bones involved. Zouari et al. published their

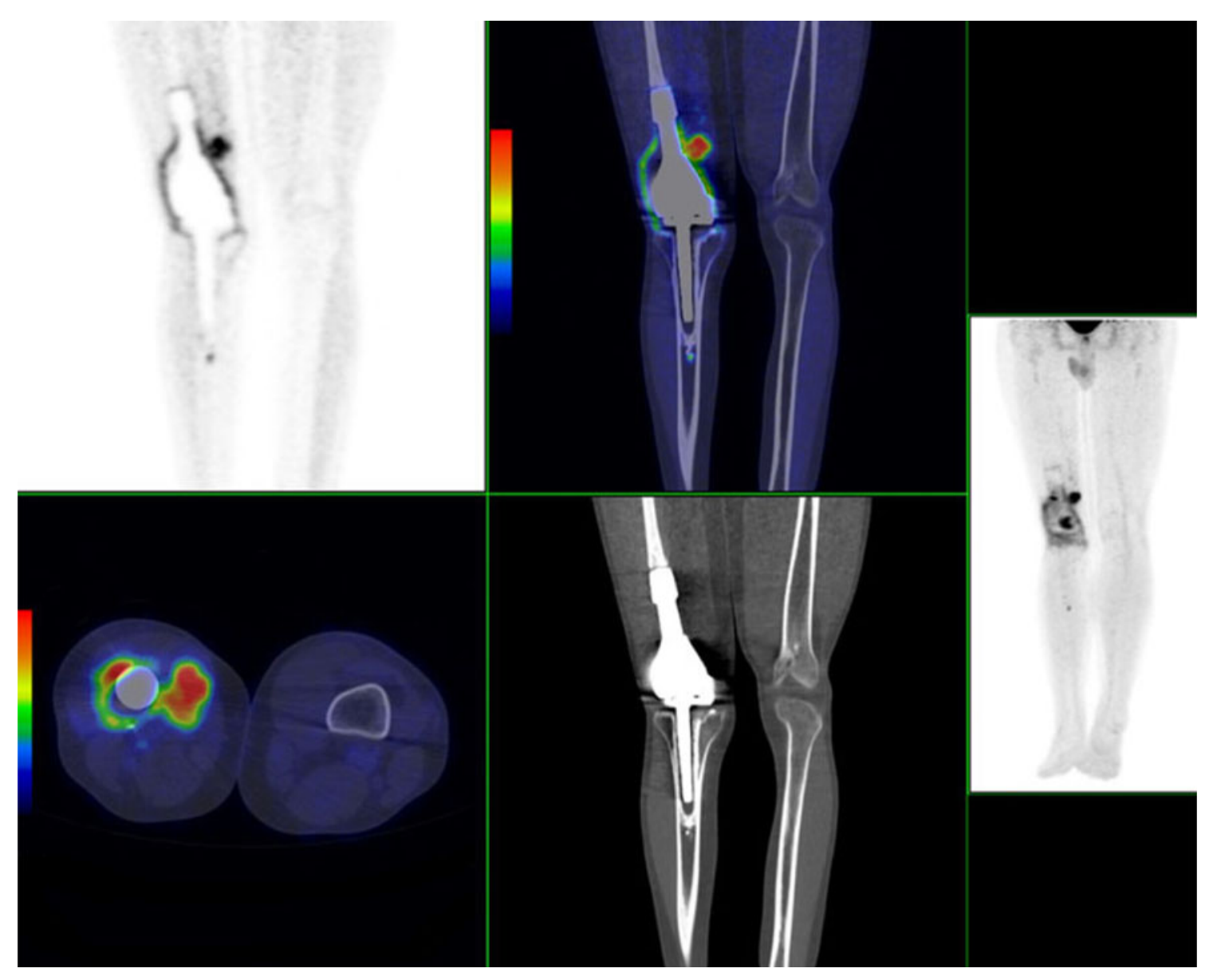

Fig. 1 Coronal FDG-PET/CT images in a patient previously treated for osteosarcoma with limb-sparing surgery and an indeterminate MRI for local recurrence due to artefact related to the metallic prosthesis. There are multiple foci of FDG avid recurrent disease around the proximal endo-prosthesis subsequently confirmed at ultrasoundguided biopsy. Figure reproduced with permission from Ref [1], Lakkaraju A et al.; article in Eur Radiol, published by Springer 
experience of 15 patients with osteoid osteomas of the hands and feet who were treated with CT-guided percutaneous laser photocoagulation [4]. In their retrospective analysis, the procedure was successful in all patients and no complications were observed, demonstrating the efficacy of CT-guided percutaneous laser photocoagulation in the treatment of osteoid-osteomas of the hands and feet.

\section{Musculoskeletal ultrasound}

Ultrasound has become one of the most rapidly expanding imaging techniques in musculoskeletal imaging. However, our knowledge and understanding of the sonographic appearance of certain anatomical structures is still evolving.

Recent anatomical studies have demonstrated that the distal biceps brachii tendon consists of two separate tendons rather than a single tendon as previously thought. Tagliafico and co-workers assessed the sonographic appearance of the distal biceps tendon bifurcation in healthy volunteers and in patients with tear of the distal biceps tendon and correlated the sonographic findings with MRI [5]. In their study, ultrasound demonstrated the two separate tendons of the distal biceps tendon in all volunteers and patients and was able to differentiate between complete tendon rupture, partial tear of the short and long head of the biceps tendon. Interestingly, isolated long head of distal biceps tendon tear clinically mimicked complete tears. In their series, ultrasound changed the management in patients with isolated tears of the long head of distal biceps tendon as these patients were treated conservatively.

Another interesting concept discussed in this journal was "tenomalacia". Khoury and Cardinal [6] assessed tendon compressibility in eight patients suffering from unilateral lateral epicondylitis and compared the findings with the asymptomatic contralateral site. They found, amongst other signs of tendinosis, increased compressibility of the common extensor tendon on the painful side compared to the asymptomatic side. The authors therefore concluded that increased compressibility indicative of tendon softening or "tenomalacia" is a new sign of common extensor tendinosis.

Recent publications demonstrate the increasingly complex and sophisticated nature of ultrasound-guided musculoskeletal interventions. De Marchi et al. retrospectively reviewed 105 patients with soft tissue masses who underwent ultrasound-guided core-needle biopsy after contrast-enhanced ultrasound [7]. They subsequently compared the histology of the biopsy with the surgically excised samples or with the expected outcome in conservatively treated patients. They reported significantly increased diagnostic sampling and accuracy in grading of soft tissue tumours using contrast-enhanced guided biopsy (Fig. 2). This study therefore reflects the increasing importance of ultrasound-guided biopsy in the diagnosis of musculoskeletal lesions.

\section{Cartilage imaging}

Osteoarthritis is a major cause of disability. Early detection and treatment of cartilage abnormalities with the aim of delaying the onset of osteoarthritis have become important and expanding topics in the musculoskeletal literature. The interest in osteoarthritis has increased due to treatment options such as microfracture, osteochondral graft transplantation and matrix-associated autologous chondrocyte
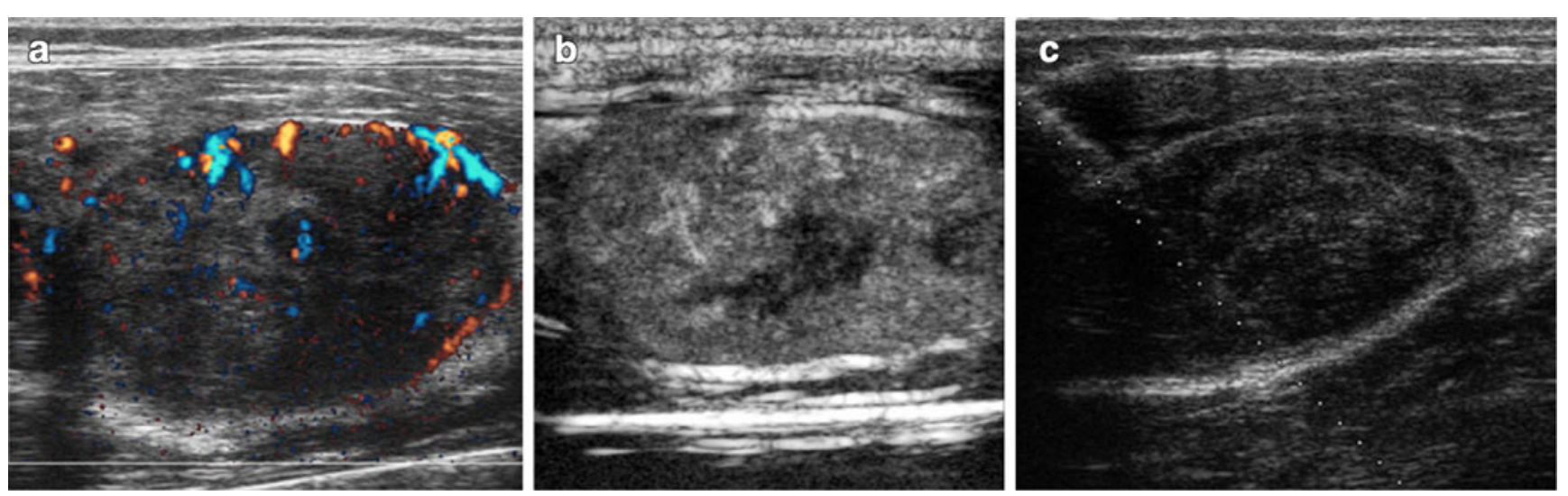

Fig. 2 a-c Mass within the quadriceps muscle: US-guided core needle biopsy $(C N B)$ is focused onto the vascularised area which is identified on contrast-enhanced ultrasound (CEUS). Histotype and grading of the biopsy revealed a high-grade leiomyosarcoma which was confirmed on the definitive surgically excised sample. a At power doppler, few and anarchic vessels are present in the peripheral region of the hypoechoic and heterogeneous mass. b CEUS shows heterogeneous enhancement and avascular areas (like "black holes"). c US-guided $\mathrm{CNB}$ is focused onto the most vascularised area, avoiding the avascular zones. Figure reproduced with permission from Ref [7], De Marchi A et al.; article in Eur Radiol, published by Springer 
implantation. These techniques demand accurate assessment of treated cartilage lesions in order to evaluate treatment success and to compare the efficacy of various surgical treatment strategies. The advent of high-field MRI has facilitated the development of novel imaging sequences such as quantitative T2-mapping, T1-delayed gadoliniumenhanced MRI of cartilage and diffusion-weighted imaging. These MRI-sequences extend beyond the morphological evaluation of cartilage allowing assessment of the biochemical ultrastructure of cartilage and may thus aid in the evaluation of cartilage tissue maturation after repair. Trattnig et al. published an excellent and in-depth review on this burgeoning topic in this journal which can only be commended [8] (Fig. 3).

3.0-Tesla MRI is increasingly used in the assessment of the knee due to the increased signal-to-noise ratio when compared to lower field strengths. However, few studies have evaluated the diagnostic efficacy of 3.0-T MRIimaging of the knee compared to $1.5 \mathrm{~T}$. Schoth et al. evaluated 20 knees imaged at 1.5 and $3.0 \mathrm{~T}$ [9]. They found that imaging at $3.0 \mathrm{~T}$ was faster and resulted in improved subjective visibility of smaller structures such as the transverse ligament and the articular cartilage.

In times of anticipated decreased public sector spending, the cost effectiveness of radiological investigations is likely to become more important than ever. Oei et al. assessed the cost effectiveness of selective short MRI in patients with acute knee injury in the absence of a fracture on radiography [10]. Patients were randomized between radiography only and radiography plus MRI. The authors found that mean total costs were lowest for the selective MRI-group and quality of life proxies (e.g. time off work) were better in the MRI group. These findings emphasize the cost-effectiveness of MRI in acute knee injury in the absence of radiographic fractures. Moreover, the findings may represent a strong argument for the expansion of musculoskeletal radiology services despite anticipated cutbacks in public sector spending.

\section{Imaging of myeloma, bone marrow and skeletal metastases}

Whole-body MRI has been proven to be the most sensitive and specific imaging investigation in the staging of multiple myeloma. However, relatively long acquisition times may represent an obstacle for the introduction of whole body MRI in the staging of multiple myeloma in daily clinical practice. Novel MRI-techniques such continuous table movement represent promising techniques which may decrease overall examination time. Weckbach et al. assessed a whole-body continuous table movement (CTM) MR protocol in 18 patients with multiple myeloma and compared it to a step-by-step whole-body MR protocol [11]. The authors found significantly reduced imaging time with the CTM-protocol when compared to the step-by-step protocol. Furthermore, depiction of bone marrow and soft tissue lesions was identical (Fig. 4). However, vertebral fractures were not detected with the CTM-protocol whilst the step-by-step protocol depicted vertebral fractures in two patients. The authors therefore concluded that although patient throughput is increased with the CTM-protocol, the protocol could not be safely used in daily clinical practice without acquisition of an additional sequence. Nevertheless, further developments in MRI-sequences may overcome this obstacle and facilitate the introduction of whole-body MRI for the staging of multiple myeloma in daily practice.

\section{Vertebroplasty and other spinal interventions}

Despite the recent controversy surrounding the efficacy of vertebroplasty in the treatment of osteoporotic fractures, vertebroplasty remains a widely used treatment option in patients who do not respond to conservative therapy [1216]. Numerous cohort studies on the efficacy of vertebroplasty have been published amongst them the paper by Masala et al. [17]. They evaluated the effectiveness of
Fig. 3 Sagittal intravenously enhanced T1-dGEMRIC maps of a patient 36 months after matrix-associated autologous chondrocyte implantation $(M A C I)$ of the medial femoral condyle $(M F C)$ (a) and a patient 36 months after microfracture (MFX) of the MFC (b). Figure reproduced with permission from Ref [8], Trattnig $\mathrm{S}$ et al.; article in Eur Radiol, published by Springer
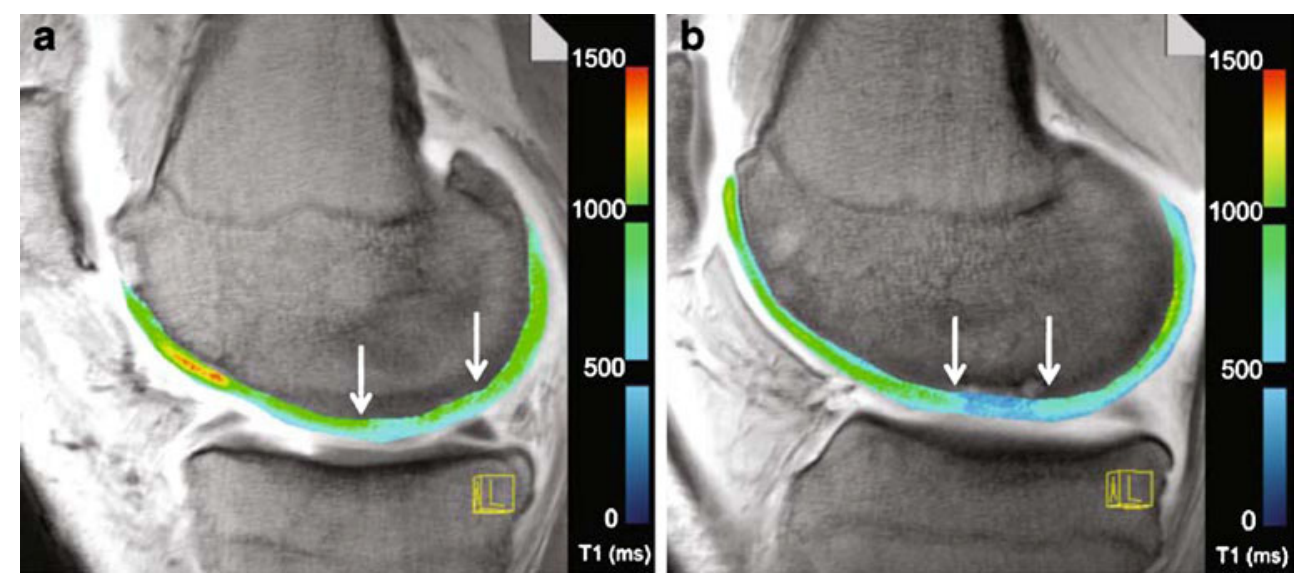

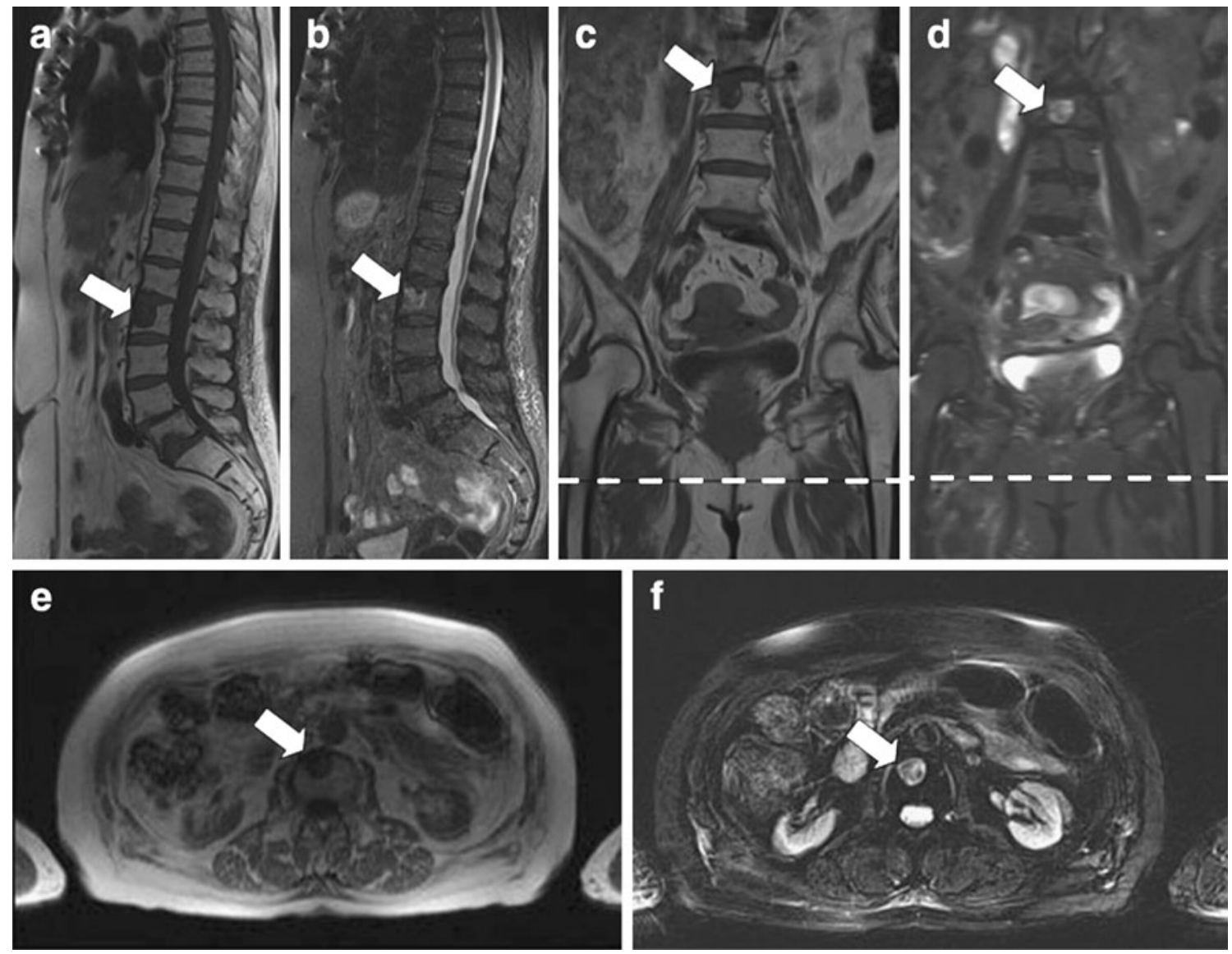

Fig. 4 A 74-year-old female patient (Durie and Salmon stage I) with a focal lesion (arrow) larger than $1 \mathrm{~cm}$ in L2. a-d Standard protocol. a Sagittal T1-w sequence, b sagittal STIR sequence, c coronal T1-w sequence, d coronal STIR sequence. e-f CTM

protocol. e T1-w GRE sequence, f T2-w fs BLADE sequence. Figure reproduced with permission from Ref [11], Weckbach S et al.; article in Eur Radiol, published by Springer

vertebroplasty in 624 patients with 1,253 compression fractures who underwent vertebroplasty for a number of indications. The authors reported a significant reduction in pain at 1 week, 3, 6 and 12 months after treatment. However, the role of vertebroplasty in the treatment of osteoporotic fractures has been called into question recently by two well publicised randomized controlled trials published in the New England journal of medicine [12, 13]. Although these randomized controlled trials were heavily criticized in the radiological literature because of various reasons such as patient selection and the set up of the sham procedure in the control group, they remain the only level 1 evidence available to date. Results from other randomized controlled trials with a more stringent patient selection such as VERTOS II are therefore eagerly awaited in order to establish the efficacy of vertebroplasty in the treatment of osteoporotic fractures [18].

Increasingly, navigation and tracking systems are being evaluated for spinal interventional procedures which allow more accurate needle placement. Spinal phantom-and animal-

studies using navigation systems for facet joint injections and intervertebral disc punctures have been promising [19]. However, further clinical studies are required to assess the benefit of these novel techniques in clinical practice.

\section{Imaging of osteoporosis}

It is essential to diagnose osteoporotic fractures accurately as pre-existing osteoporotic fractures increase the risk of future fractures significantly leading to increased morbidity and mortality. Multidetector CT (MD-CT) provides thinsection images of the chest and abdomen therefore allowing high-resolution sagittal reconstructions. In daily clinical practice, MD-CT is routinely used in a wide variety of patients for clinical indications which are unrelated to osteoporotic fractures. Müller et al. assessed, amongst other questions, osteoporotic vertebral fractures on sagittal reformations of routine MD-CT-scans of the chest and/or abdomen in 112 postmenopausal women [20]. The authors 
discovered osteoporotic fractures in 27 patients of which none were mentioned in the radiology report; only six of these osteoporotic fractures were identifiable on routine axial CT images. The authors therefore concluded that sagittal reformations increase the depiction of osteoporotic fractures when compared to axial images and should therefore be obtained in all patients at risk of osteoporosis who undergo routine MDCT.

\section{Imaging of the shoulder}

Both ultrasound and MRI are frequently used imaging modalities in the assessment of rotator cuff tears. However the role of ultrasound in comparison to MRI in the diagnosis of rotator cuff tears remains equivocal. Rutten et al. retrospectively evaluated the need for additional MRI/ MRA in 5,216 patients who underwent ultrasound of the shoulder for suspected rotator cuff tear [21]. Furthermore, they compared the accuracy of ultrasound and MRI/MRA for the detection of partial-and full-thickness tears with surgical findings. They found that MRI or MRA of the shoulder in addition to ultrasound was performed in only $5.2 \%$. Both ultrasound and MRI depicted partial and fullthickness tear with a high accuracy. MRI was of additional value in the detection of intra-articular lesions. The authors therefore concluded that after ultrasound of the shoulder by an experienced radiologist, MRI offers little additional value in the detection of rotator cuff tears.

\section{Conclusion}

This review demonstrates that research in musculoskeletal imaging is alive and well. Recent years have shown increased research activity in musculoskeletal oncology, expansion of musculoskeletal ultrasound and exciting advances in novel imaging techniques. $3 \mathrm{~T}$ imaging is now firmly established in clinical practise and the availability and use of this higher field strength is only likely to increase. The future is also likely to see an increasing role of PET/CT and other forms of molecular imaging and the introduction of whole-body MRI into daily clinical practice. We also look forward to ongoing research about the role of novel imaging techniques and the clarification of the role vertebroplasty in the treatment of osteoporotic fractures.

\section{References}

1. Lakkaraju A, Patel CN, Bradley KM, Scarsbrook AF (2010) PET/ CT in primary musculoskeletal tumours: a step forward. Eur Radiol 20:2959-2972, Epub 2010 Jun 25
2. Alyas F, Saifuddin A (2008) Fluid-fluid levels in bone neoplasms: variation of $\mathrm{T} 1$-weighted signal intensity of the superior to inferior layers-diagnostic significance on magnetic resonance imaging. Eur Radiol 18:2642-2651

3. Singh J, James SL, Kroon HM, Woertler K, Anderson SE, Jundt G, Davies AM (2009) Tumour and tumour-like lesions of the patella-a multicentre experience. Eur Radiol 19:701-712

4. Zouari L, Bousson V, Hamzé B, Roulot E, Roqueplan F, Laredo JD (2008) CT-guided percutaneous laser photocoagulation of osteoid osteomas of the hands and feet. Eur Radiol 18:2635-2641

5. Tagliafico A, Michaud J, Capaccio E, Derchi LE, Martinoli C (2010) Ultrasound demonstration of distal biceps tendon bifurcation: normal and abnormal findings. Eur Radiol 20:202-208

6. Khoury V, Cardinal E (2009) "Tenomalacia": a new sonographic sign of tendinopathy? Eur Radiol 19:144-146

7. De Marchi A, Brach Del Prever EM, Linari A, Pozza S, Verga L, Albertini U, Forni M, Gino GC, Comandone A, Brach Del Prever AM, Piana R, Faletti C, Piedmont Sarcoma Group (2010) Accuracy of core-needle biopsy after contrast-enhanced ultrasound in soft-tissue tumours. Eur Radiol 20:2740-2748, Epub 2010 Jun 27

8. Trattnig S, Domayer S, Welsch GW, Mosher T, Eckstein F (2009) MR imaging of cartilage and its repair in the knee-a review. Eur Radiol 19:1582-1594

9. Schoth F, Kraemer N, Niendorf T, Hohl C, Gunther RW (2008) Comparison of image quality in magnetic resonance imaging of the knee at 1.5 and 3.0 Tesla using 32-channel receiver coils. Eur Radiol 18:2258-2264

10. Oei EH, Nikken JJ, Ginai AZ, Krestin GP, Verhaar JA, van Vugt AB, Hunink MG (2009) Costs and effectiveness of a brief MRI examination of patients with acute knee injury. Eur Radiol 19:409-418

11. Weckbach S, Michaely HJ, Stemmer A, Schoenberg SO, Dinter DJ (2010) Comparison of a new whole-body continuous-tablemovement protocol versus a standard whole-body MR protocol for the assessment of multiple myeloma. Eur Radiol 20:29072916, Epub 2010 Jun 24

12. Buchbinder R, Osborne RH, Ebeling PR, Wark JD, Mitchell P, Wriedt C, Graves S, Staples MP, Murphy B (2009) A randomized trial of vertebroplasty for painful osteoporotic vertebral fractures. N Engl J Med 361:557-568

13. Kallmes DF, Comstock BA, Heagerty PJ, Turner JA, Wilson DJ, Diamond TH, Edwards R, Gray LA, Stout L, Owen S, Hollingworth W, Ghdoke B, Annesley-Williams DJ, Ralston SH, Jarvik JG (2009) A randomized trial of vertebroplasty for osteoporotic spinal fractures. N Engl J Med 361:569-579

14. Kallmes DF, Jarvik JG, Osborne RH, Comstock BA, Staples MP, Heagerty PJ, Turner JA, Buchbinder R (2010) Clinical utility of vertebroplasty: elevating the evidence. Radiology 255:675-680

15. Baerlocher MO, Munk PL, Liu DM, Tomlinson G, Badii M, Kee ST, Loh CT, Hardy BW, Murphy KJ (2010) Clinical utility of vertebroplasty: need for better evidence. Radiology 255:669-674

16. Noonan P (2009) Randomized vertebroplasty trials: bad news or sham news? AJNR Am J Neuroradiol 30:1808-1809

17. Masala S, Mastrangeli R, Petrella MC, Massari F, Ursone A, Simonetti G (2009) Percutaneous vertebroplasty in 1, 253 levels: results and long-term effectiveness in a single centre. Eur Radiol 19:165-171

18. Klazen CA, Verhaar HJ, Lampmann LE, Juttmann JR, Blonk MC, Jansen FH, Tielbeek AV, Schoemaker MC, Buskens E, van der Graaf Y, Janssens X, Fransen H, van Everdingen KJ, Muller AF, Mali WP, Lohle PN (2007) VERTOS II: percutaneous vertebroplasty versus conservative therapy in patients with painful osteoporotic vertebral compression fractures; rationale, objectives and design of a multicenter randomized controlled trial. Trials $8: 33$ 
19. Bruners P, Penzkofer T, Nagel M, Elfring R, Gronloh N, SchmitzRode T, Günther RW, Mahnken AH (2009) Electromagnetic tracking for CT-guided spine interventions: phantom, ex-vivo and in-vivo results. Eur Radiol 19:990-994

20. Müller D, Bauer JS, Zeile M, Rummeny EJ, Link TM (2008)

Significance of sagittal reformations in routine thoracic and abdominal multislice CT studies for detecting osteoporotic fractures and other spine abnormalities. Eur Radiol 18:16961702

21. Rutten MJ, Spaargaren GJ, van Loon T, de Waal Malefijt MC, Kiemeney LA, Jager GJ (2010) Detection of rotator cuff tears: the value of MRI following ultrasound. Eur Radiol 20:450-457 